\title{
Micro-Laser-Assisted Machining: The Future of Manufacturing Ceramics and Semiconductors
}

\author{
Deepak Ravindra* and John Patten \\ Micro-LAM Technologies LLC, 4950 W. Dickman Rd., Suite AA-1, Battle Creek, MI 49009, USA
}

(Received January 22, 2014; accepted February 24, 2014)

Key words: $\quad \mu$-LAM, single-point diamond turning, ductile-mode machining, micro-laser-assisted machining, high-pressure phase transformation

Ceramics and semiconductors are hard, strong, inert and lightweight. They also have good optical properties, wide energy bandgap and high maximum current density. This combination of properties makes them ideal candidates for tribological, semiconductor, micro-electromechanical systems (MEMS) and optoelectronic applications. Manufacturing these materials without causing surface and subsurface damage is extremely challenging owing to their high hardness, brittle characteristics and poor machinability. However, ductile regime machining of these materials is possible owing to the high-pressure phase transformation occurring in the materials caused by the high compressive stresses induced by the single-point diamond tool tip. In this study, to further augment the ductile response of the machined material, single-point scratch tests and diamond turning are coupled with the $\mu$-LAM technique. The high-pressure phase is preferentially heated and thermally softened by using concentrated energy sources (i.e., laser beams) to enhance the ductile response of the material. The focus here is to develop an efficient manufacturing technique to improve the surface quality of ceramics and semiconductors to be used as optical devices (mirrors and windows). Machining parameters such as the depth of cut, feed, cutting speed and laser power are optimized to make the manufacturing process shorter and cost-effective.

\section{Introduction}

Various materials including metals and alloys, ceramics, glasses, semiconductors and composites are manufactured to meet service requirements to the given geometry, accuracy, finish and surface integrity. ${ }^{(1)}$ Metals and alloys in general are easier to machine in the ductile regime because of their high fracture toughness, low hardness, nondirectional bonding, low porosity, large strain to fracture and high impact energy. Nonmetals, on the other hand, such as ceramics and semiconductors, are characterized by

*Corresponding author: e-mail: deepak.ravindra@micro-lam.com 
covalent or ionic bonding, limited slip systems for plastic deformation, high hardness and low fracture toughness. ${ }^{(2)}$ This is due to the major differences indicating that ceramics and semiconductors are "difficult-to-machine" nominally brittle materials.

Dramatic advances have occurred in ceramics technology since the mid-1990s. ${ }^{(3)}$ New and improved ceramics and semiconductors that have much higher strength and toughness than previous ceramics are now available. ${ }^{(4)}$ Major research and development has been accomplished over the past 20-30 years to increase the capability of ceramics and semiconductors for thermal, wear, optical, corrosion and structural applications. These intrinsic properties and developments make advanced ceramics and semiconductors exceptional candidates in harsh environments such as high-temperature, strong-radiation, and corrosive and abrasive media. Some of the application fields utilizing advanced ceramics and semiconductors include automobile, aerospace, petroleum, nuclear, military and biological/medical industries. Manufacturing these materials is extremely challenging owing to their high hardness, brittle characteristics and poor machinability. Severe fracture can result when trying to machine ceramics and semiconductors owing to their low fracture toughness. However, from past experience, it has been proven that ductile regime machining of brittle materials is possible. ${ }^{(5)}$ Some studies indicate that ductility is related to the high-pressure phase transformation (HPPT) that occurs when these materials are subjected to high hydrostatic pressures and shear stresses. ${ }^{(6)}$

Current limitations for brittle material machining include the high cost of processing and low product reliability. The cost is mainly due to expensive tools that wear out rapidly, long machining time, low production rate and the manufacturing of satisfactory surface roughness, figure and form. The low product reliability is primarily due to the occurrence of surface/subsurface damage, i.e., cracks, and brittle fracture. In order to develop a suitable process, ductile regime machining, considered as one of the satisfactory precision machining techniques, has been continuously studied over the last two decades. ${ }^{(7-15)}$ Laser-assisted micro/nanomachining is another important development in this direction. ${ }^{(16,17)}$

Past research has demonstrated that ductile regime machining of these materials is possible owing to the HPPT occurring in the materials caused by the high compressive and shear stresses induced by the single-point diamond tool tip. ${ }^{(18)}$ To further augment the ductile response of these materials, traditional scratch/single-point diamond cutting tests are coupled with a micro-laser-assisted machining ( $\mu$-LAM) technique. ${ }^{(19)}$

A schematic of the basic underlying concept of the $\mu$-LAM process is shown in Fig. 1. An infrared (IR) beam (passed through a transparent diamond tool) is typically used in this process as $\mathrm{Si}$ and $\mathrm{SiC}$ are almost transparent at this wavelength. This allows the laser beam to be absorbed only by the high-pressure metallic phases resulting in enhanced ductility and reduced hardness during the material removal process. This hybrid configuration thermally heats and softens the HPPT material, making it more ductile, and increasing the critical depth of cut (DoC) [larger ductile-to-brittle transition (DBT) depth] in ductile regime machining, resulting in a higher material removal rate. Also, the reduction in the hardness of the contact angle (high-pressure phase) significantly contributes to prolonging the tool life.

In this paper, we discuss results from experimental work carried out to determine 


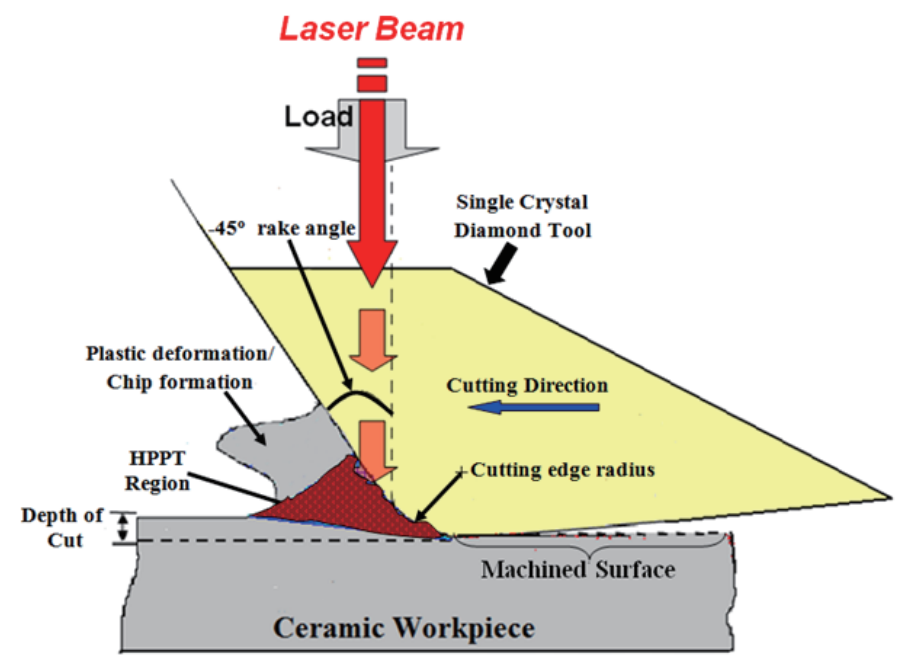

Fig. 1. (Color online) Schematic cross section of $\mu$-LAM process.

the DBT depths of several ceramics and semiconductors of interest. The effect of laser heating on the DBT depth is compared for three polytypes of silicon carbide ( $\mathrm{SiC}$ ), silicon ( $\mathrm{Si})$, and sapphire.

\section{Experimental Methods}

Scratch tests are carried out using a $1-\mathrm{mm}$-nose-radius tool $\left(-45^{\circ}\right.$ rake and $5^{\circ}$ clearance angle) to determine the DBT depth of the materials. Scratch tests are chosen to be the principal method of investigation in this study as they are a better candidate for evaluating machining conditions than indenting because the mechanics during scratching are more applicable to machining processes such as single point diamond turning (SPDT). The scratch tests are performed with increasing load (thrust force) to produce the ductile, DBT and brittle regimes. Once the scratch tests are completed, microscopy images and force data are obtained, and profilometer analyses are carried out and correlated in order to determine the DBT depth (with the corresponding cutting and thrust forces).

The scratch tests are performed on a universal material tester (UMT), which is produced by the BRUKER Center for Tribology Research Inc. (CETR). This equipment is developed to perform comprehensive micromechanical tests of coatings and materials at the microscale with a nanometric resolution. This system facilitates cutting at a speed as low as $1 \mu \mathrm{m} / \mathrm{s}$ at nanometric cutting depths. Unlike commercial lathes, the UMT is a load-controlled device (versus depth-controlled) where the required thrust force/load $\left(F_{z}\right)$ is applied by the user to obtain the desired depth of cut (based on the tool geometry and workpiece material properties). The unit is equipped with a dual-axis load cell that is capable of constantly monitoring the thrust and cutting forces $\left(F_{x}\right)$, which are obtained as an output parameter from the cutting experiment. A typical scratch test setup on the 
UMT is shown in Fig. 2. A 1-mm-nose-radius single-crystal diamond cutting tool $\left(-45^{\circ}\right.$ rake and $5^{\circ}$ clearance), similar to that used in SPDT experiments, is used for this set of experiments. The reason for using an actual cutting tool in these tests is to establish DBT depths that can be used during actual SPDT operations. A stylus generates a more aggressive cut, but the depth and loads are not applicable during SPDT. Also, using similar tool geometries generates brittle fracture similar to that in an actual SPDT operation, further enabling the prediction of fracture behavior in these materials during machining.

The scratch test parameters are summarized in Table 1 for all five materials experimented on. The applied load range is chosen on the basis of some preliminary results where the material is seen to exhibit both ductile and brittle characteristics (with a DBT zone). The DBT depths of a total of five materials of interest are also determined with laser heating. The DBT depths obtained with laser heating are then compared with the DBT depths at room temperature to study the ductile response of the material due to thermal softening. Thermal softening of the material via laser heating has been

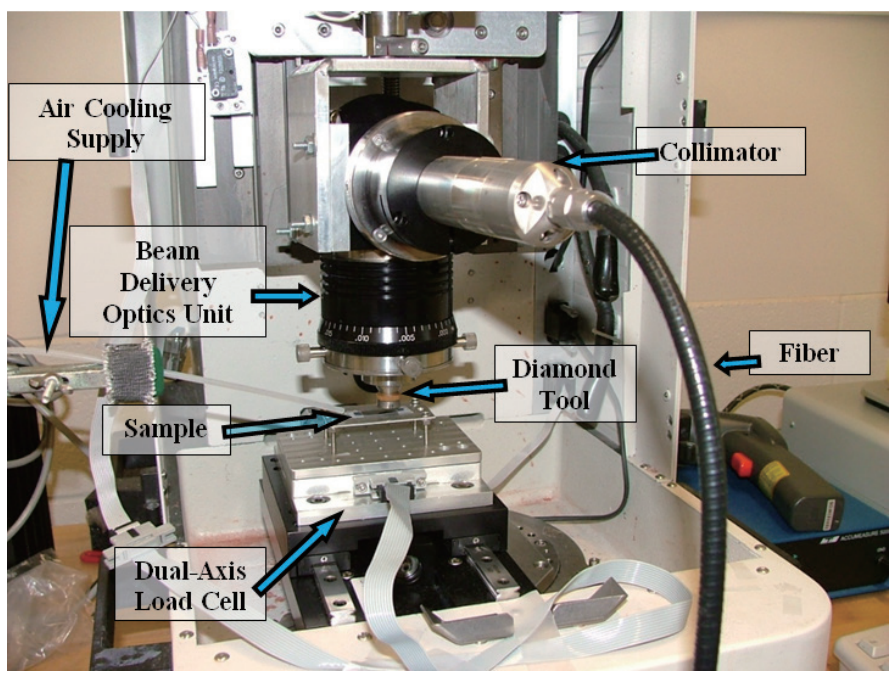

Fig. 2. (Color online) High-powered $\mu$-LAM scratch test setup on UMT.

Table 1

Scratch test parameters for experimented materials.

\begin{tabular}{lccrc}
\hline Material & Form & Hardness & Applied load range & Heating condition \\
\hline $4 \mathrm{H}-\mathrm{SiC}$ & Single crystal & $26 \mathrm{GPa}$ & $70-200 \mathrm{mN}$ & With and without laser \\
$6 \mathrm{H}-\mathrm{SiC}$ & Single crystal & $26 \mathrm{GPa}$ & $70-200 \mathrm{mN}$ & With and without laser \\
$3 \mathrm{C}-\mathrm{SiC}$ & Polycrystalline & $27 \mathrm{GPa}$ & $200-700 \mathrm{mN}$ & With and without laser \\
Silicon & Single crystal & $12 \mathrm{GPa}$ & $50-300 \mathrm{mN}$ & With and without laser \\
Sapphire & Single crystal & $23 \mathrm{GPa}$ & $400-800 \mathrm{mN}$ & With and without laser \\
\hline
\end{tabular}


proven to be beneficial in the material removal process by enhancing the ductility of brittle materials. In order to preferentially heat the material, traditional scratch tests are coupled with the $\mu$-LAM technique. All cutting processes reported here are performed at a constant speed of $1 \mu \mathrm{m} / \mathrm{s}$.

The scratch tests are then performed by optical microscopy to detect the onset of brittle behavior; white light interferometry for depth profile measurements and cutting force analyses are very sensitive to brittle activity. The final part of the DBT analysis is relating the DBT depths and forces to a few crucial material mechanical properties that are believed to affect the ductile response during material removal.

\section{Results and Discussion}

As the experimental technique is unchanged for all materials tested, sapphire will be used for discussion purposes to better understand the testing process.

\subsection{Force data}

Unstable forces (cutting and thrust) are usually good indicators of brittle fracture in the material removal process. The cutting force is extremely sensitive to fracture and brittle activities, as it generally occurs along the cutting direction. This is clearly illustrated in Fig. 3, where the effect of brittle fracture along the cut is clearly seen in the cutting force data. During the material removal process using a single-point diamond tool, the cutting force can vary owing to numerous reasons, such as surface roughness,

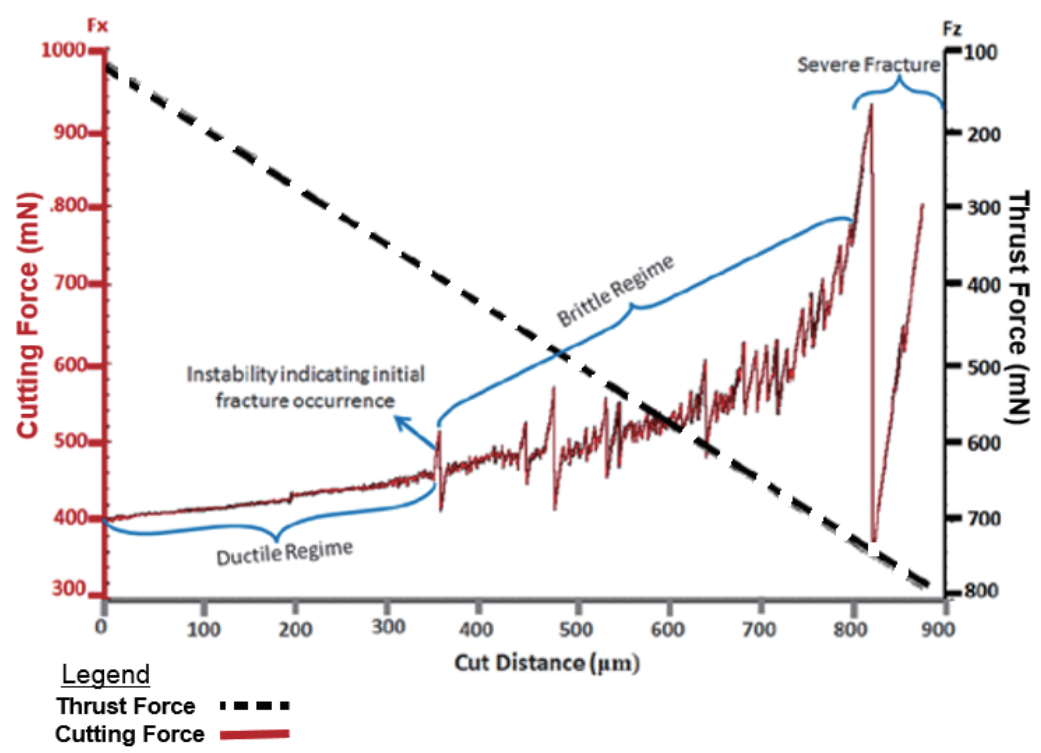

Fig. 3. (Color online) Force data correlated to ductile and brittle modes for cut with no laser heating. 
debris from machining, lack of lubrication/slurry, uneven tool wear, and external vibration. In general, the lower the cutting force, the better the overall performance of the machining process (lower cutting force is desired). A lower cutting force is also desired as it significantly extends the tool life during machining.

In Fig. 3 the force data of a scratch test performed on sapphire is shown. A linear increase in load from 500 to $1100 \mathrm{mN}$ is used to exhibit both ductile and brittle modes of material removal. It is clearly observed that the cutting force is very sensitive to brittle fracture and results in instability when fracture occurs. The initial instability observed at approximately $430 \mathrm{mN}$ in the presence of the cutting force is generally the point at which the material starts to exhibit brittle behavior. Beyond this point, the standard deviation of the cutting force increases significantly owing to the uncontrolled material removal process. Monitoring the cutting force during the cutting process is also an effective in situ monitoring method to avoid brittle-mode machining.

In addition to a single cut with increasing load, multiple cuts with constant loads were also examined. A series of cutting processes are carried out to quantify the correlation between the applied load, laser heating (power), depth of cut, ductile response and cutting force. Table 2 summarizes the scratch test parameters and results obtained at a constant cutting speed of $1 \mu \mathrm{m} / \mathrm{s}$.

\subsection{Optical microscopy}

Analyzing the cuts using optical microscopy is an extremely time-efficient and straightforward method of detecting brittle behavior on the machined surface. By using the appropriate magnification, optical microscopy is used to analyze the materialremoved surfaces. A brittle-mode material removal process can be distinctly identified, as it leaves a poor surface finish owing to the cracks and voids/divots formed during

Table 2

Scratch test parameters at a constant cutting speed.

\begin{tabular}{lccc}
\hline & Applied load $(\mathrm{mN})$ & Depth range $(\mathrm{nm})$ & Cutting force $(\mathrm{mN})$ \\
\hline \multirow{4}{*}{ No laser } & 350 & $55-60$ & 210 \\
& 400 & $65-68$ & 240 \\
& 450 & $73-77$ & 275 \\
& 500 & $80-84$ & 310 \\
& 550 & $87-91$ & 345 \\
& 600 & $102-108$ & 385 \\
$10 \mathrm{~W}$ & 650 & $120-125$ & 430 \\
& 700 & $\mathrm{~N} / \mathrm{A}($ Brittle $)$ & N/A (Brittle) \\
\hline \multirow{3}{*}{$20 \mathrm{~W}$} & 400 & $75-82$ & 255 \\
& 500 & $100-105$ & 335 \\
& 600 & $135-140$ & 410 \\
\hline
\end{tabular}


cutting. Figure 4 shows a series of cutting processes performed on single-crystal sapphire at different loads and laser powers.

In Figs. 4(a)-4(c), it is clearly seen that the cuts under all heating conditions at 500 $\mathrm{mN}$ exhibit a fully ductile behavior as the DBT threshold has not been reached for either condition. Although all three conditions exhibit ductile behavior, the depths of cuts with laser heating are greater than those without laser heating $(\sim 25-30 \%$ greater). The cuts in Figs. 4(d)-4(f) start to exhibit brittle behavior indicating that the DBT depths have been reached for each condition. In general, the brittle behavior is more defined along the center of the cut, as this is the deepest region of the cut (owing to the $1 \mathrm{~mm}$ tool nose radius). Beyond the DBT depth, the cuts tend to be mostly brittle. The cutting processes performed in the partial or fully brittle regime leave a poorly defined cut edge with uncontrolled cracking. In brittle-mode machining, once a crack reaches the edge of a cut (outside the compressive zone), it could encounter a tensile field and propagate beyond the scratch edges.

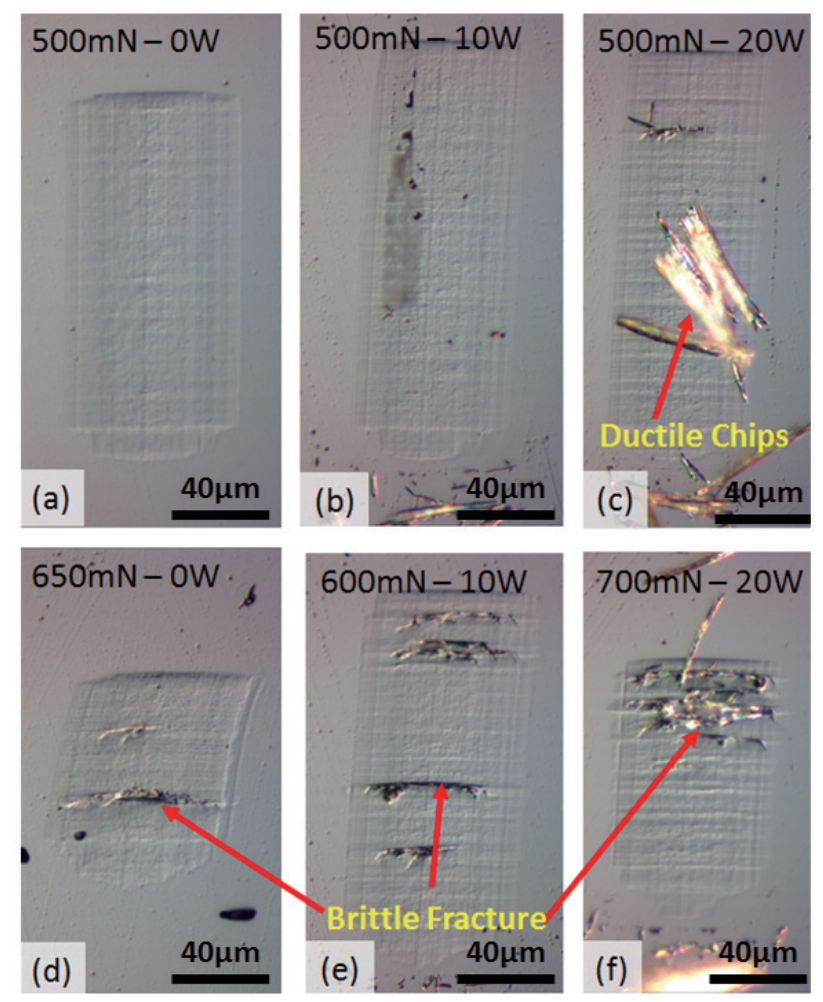

Fig. 4. (Color online) Cuts at different loads and laser powers: (a-c) all cuts showing fully ductile behavior and (d-f) DBT. 


\subsection{White light interferometry}

In this study, white light interferometry is used to measure the depth of cut and analyze the cut profile. In some materials, the presence of grains makes it challenging to differentiate a ductile out from a partially brittle cut. However, this is not observed in this material as single-crystal sapphire was used.

Figures 5 and 6 show an example for both cases; a fully ductile cut is shown in Fig. 5 and a partially brittle cut is shown in Fig. 6.

Figure 5 shows the well-defined tool imprint represented by the cross-sectional profile for the cut formed on sapphire. There are no signs of material fracture observed in the groove of a perfectly ductile cut.

Figure 6 indicates the DBT of the sapphire sample where the well-defined tool imprint is not evident at the tip of the groove. The DBT depth measured in this particular scan is approximately $400 \mathrm{~nm}$ for the cut obtained at $20 \mathrm{~W}$ laser power at $700 \mathrm{mN}$.

Figure 5 shows a typical three-dimensional (2-D) cross section of a perfectly ductile
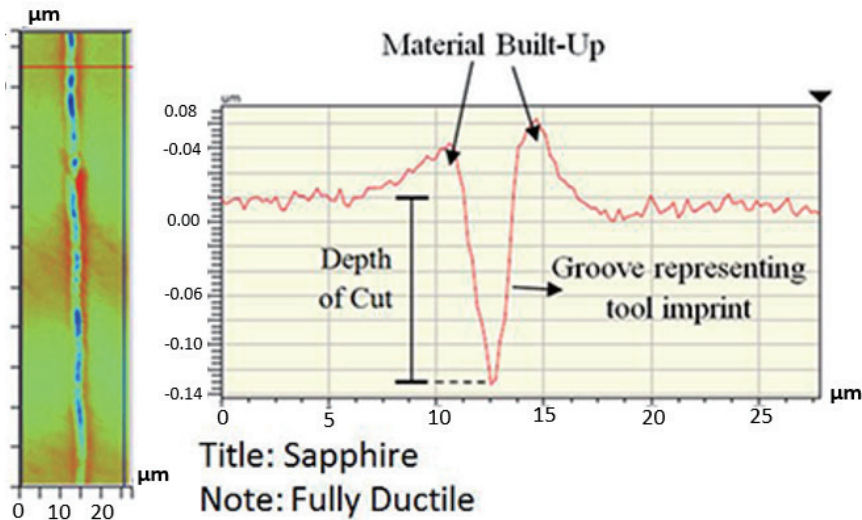

Title: Sapphire

Note: Fully Ductile

Fig. 5. (Color online) Surface profile of a fully ductile cut.

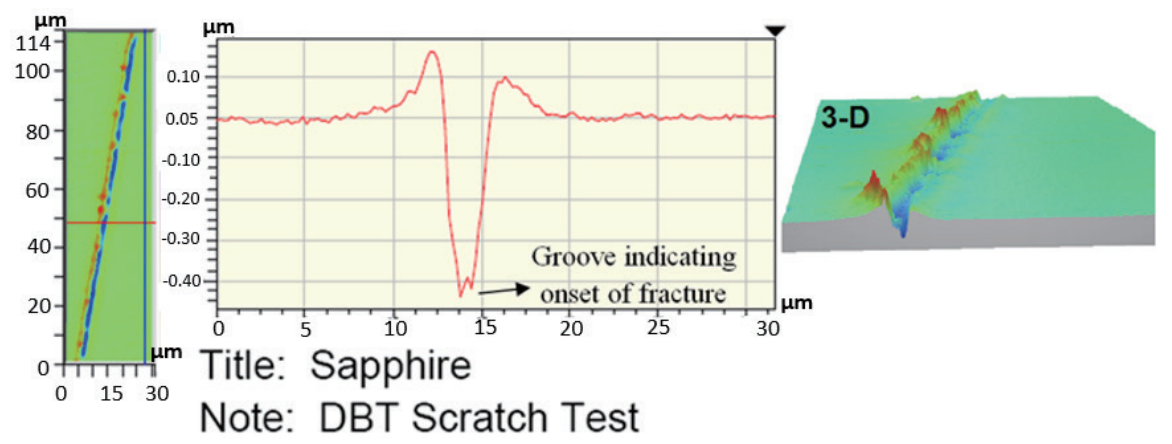

Fig. 6. (Color online) Surface profile above indicating the DBT. 
region along with a 2-D scratch profile. Figure 6 shows the cross section at the DBT zone (the point at which fracture is first identified) in sapphire. The defined feature of the tool imprint seen in Fig. 5 is no longer visible in Fig. 6. In this study, 2-D cross-sectional profiling of the cuts is analyzed at every point along the cut to obtain depths of cuts at various points. It is important to perform a three-dimensional analysis along the cut (in this case, white light interferometry was chosen as the method of analysis) as the onset of fracture could occur beneath the material-removed surface (subsurface) before appearing on the surface.

\section{Summary of Results for All Materials}

In this section, we aim to compare the DBT depths obtained with and without laser heating for the five selected materials of interest (as shown in Fig. 7). Table 3 shows the DBT parameters obtained with and without laser heating.

It is seen that all cuts obtained with laser heating are able to withstand higher applied loads without exhibiting brittle fracture. This is the key to increasing the DBT depth of the material. The effect of laser heating is evident where greater loads can be applied, hence significantly increasing the DBT for all five materials. This is highlighted in Fig. 7 where the DBT depth is compared for all five materials and both conditions, that is, with and without laser heating.

The most significant increase in DBT depth $(\sim 176 \%)$ is observed in the $3 \mathrm{C}$ polycrystalline $\mathrm{SiC}$. This is believed to be due to the following two factors: (1) the $3 \mathrm{C}$ absorbs more than $95 \%$ laser radiation at these wavelengths and (2) the polycrystalline cubic SiC structure has the highest fracture toughness (among these five materials), which could be a reason for the reduced brittleness in the atmospheric phase observed during laser heating. Although the DBT is shown to occur at different applied loads,

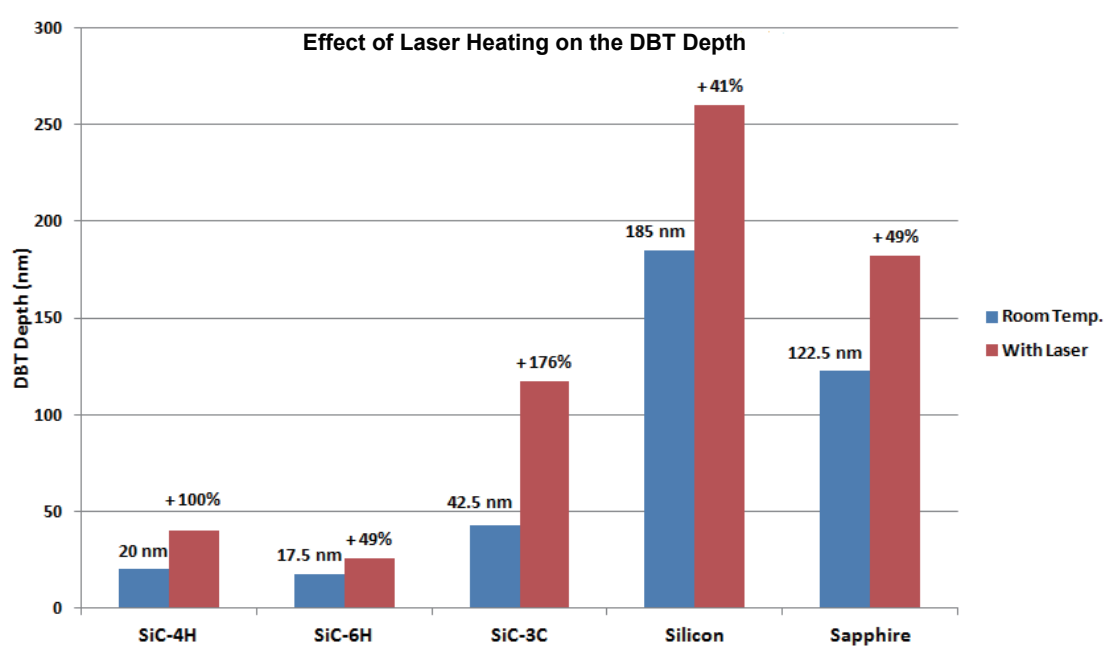

Fig. 7. (Color online) Comparison of DBT depths obtained with and without laser heating. 
Table 3

DBT parameters for materials experimented with and without laser heating.

\begin{tabular}{lrccccc}
\hline & \multicolumn{3}{c}{ Room temperature } & \multicolumn{3}{c}{ Laser heating } \\
\hline Material & $\begin{array}{c}\text { DBT } \\
\text { load }^{*}\end{array}$ & $\begin{array}{c}\text { DBT cutting } \\
\text { force* }\end{array}$ & $\begin{array}{c}\text { Avg. DBT } \\
\text { depth }^{*}\end{array}$ & $\begin{array}{c}\text { DBT } \\
\text { load }^{*}\end{array}$ & $\begin{array}{c}\text { DBT cutting } \\
\text { force }\end{array}$ & $\begin{array}{c}\text { Avg. DBT } \\
\text { depth }^{*}\end{array}$ \\
\hline $4 \mathrm{H}-\mathrm{SiC}$ & $85 \mathrm{mN}$ & $31 \mathrm{mN}$ & $20.0 \mathrm{~nm}$ & $120 \mathrm{mN}$ & $46 \mathrm{mN}$ & $40.0 \mathrm{~nm}$ \\
$6 \mathrm{H}-\mathrm{SiC}$ & $120 \mathrm{mN}$ & $48 \mathrm{mN}$ & $17.5 \mathrm{~nm}$ & $140 \mathrm{mN}$ & $58 \mathrm{mN}$ & $26.0 \mathrm{~nm}$ \\
$3 \mathrm{C}-\mathrm{SiC}$ & $450 \mathrm{mN}$ & $230 \mathrm{mN}$ & $42.5 \mathrm{~nm}$ & $600 \mathrm{mN}$ & $330 \mathrm{mN}$ & $117.5 \mathrm{~nm}$ \\
Silicon & $175 \mathrm{mN}$ & $105 \mathrm{mN}$ & $185.0 \mathrm{~nm}$ & $200 \mathrm{mN}$ & $120 \mathrm{mN}$ & $260.0 \mathrm{~nm}$ \\
Sapphire & $650 \mathrm{mN}$ & $430 \mathrm{mN}$ & $122.5 \mathrm{~nm}$ & $700 \mathrm{mN}$ & $520 \mathrm{mN}$ & $182.5 \mathrm{~nm}$ \\
\hline
\end{tabular}

Note: *Measurement obtained just before the DBT (first point of fracture occurrence)

the key point here is that laser heating allows for deeper cuts before exhibiting brittle behavior ( $40-175 \%$ as shown in Fig. 7). Overall, the experimental results suggest that laser-assisted scratch tests are successful in enhancing the ductile response in all five materials.

\section{Conclusions}

Laser heating was successfully demonstrated as evidenced by the significant increase in the ductile response of five selected materials of interest (three $\mathrm{SiC}$ polytypes, $\mathrm{Si}$ and sapphire). Laser-assisted (heating, thermal softening and reduced brittleness) material removal resulted in smaller cutting forces (when comparing the relative depth-to-cutting force ratio) and a larger critical depth of cut (DBT depth). Micro-laser assisted scratch tests were successful in demonstrating the enhanced thermal softening in $\mathrm{Si}, \mathrm{SiC}$ (all three polytypes) and sapphire resulting in greater depths of cuts (when compared with similar applied loads for cuts with no laser) and greater ductile-to-brittle transition depths. Laser-assisted (heating, thermal softening and reduced brittleness) material removal resulted in greater depths of cuts at less applied thrust forces, smaller cutting forces and a larger critical depth of cut. Force analyses (thrust and cutting), optical microscopy and white light interferometry served as useful analysis (measurement and characterization) methods for detecting the enhanced ductile response and reduced brittle fracture as a result of preferential material heating. Results obtained from this study are promising in the further implementation of the $\mu$-LAM technique in machining operations such as SPDT. Lower cutting forces obtained from the $\mu$-LAM process are favorable for minimizing tool wear while machining abrasive ceramics/semiconductors such as SiC. No tool wear was detected in these tests owing to small machined areas (short track length covered by the tool); however, more testing will be performed in the future to machine a larger area for tool wear analysis. The results of this study will also benefit the manufacture of brittle materials as laser heating is proven to decrease the brittle response in ceramics and semiconductors, which can result in high productivity rates (i.e., higher material removal rates).

The results show strong evidence that the laser irradiation preferentially heats and greatly effects the high-pressure phases. Laser-assisted heating and thermal softening 
during material deformation and removal resulted in reduced brittleness and greater depths of cuts at equal applied thrust forces and lower cutting forces. The key to a successful and productive ductile-mode machining of brittle materials is understanding the material removal mechanics and optimizing the processing parameters. The findings in this research will lead to the development of improved machining technologies by utilizing the ductile-mode material removal mechanism and reduced brittle-mode material removal in the manufacture of ceramics and semiconductors.

\section{Acknowledgements}

The authors would like to thank the National Science Foundation (SBIR Phase I/IB Award \# IIP-1214772 and SBIR Phase II Award \# IIP-1330439) for funding this study. The authors would also like to thank the II-VI Foundation for their funding via the block gift program.

\section{References}

1 W. Konig, L. Crionjager, U. Dortmund, G. Spur, H. K. Tonshoff, M. Vigneau and W. J. Zdeblick: Machining of New Materials, Ann. CIRP. 39 (1990) 673.

2 D. B. Marshal, B. R. Lawn and A. G. Evans: J. Am. Ceram. Soc. 65 (1982) 561.

3 D. W. Freitag and D. W. Richerson: Oak Ridge National Laboratory, Report DOE/ORO (1998) 2076.

4 J. B. Wachtman, Jr.: Treatise Mater. Sci. Technol. 29 (1989) 150.

5 J. Patten and B. Bhatt: Proc. ASME Int. Manufacturing Science and Engineering Conference 2006 (Michigan).

6 P. S. Pizani, R. Jasinevicius, J. G. Duduch and A. J. V. Porto: J. Mater. Sci. Lett. 18 (1999) 1185.

7 P. N. Blake and R. O. Scattergood: J. Am. Ceram. Soc. 73 (1990) 949.

8 W. S. Blackley and R. O. Scattergood: J. Eng. Ind. 116 (1994) 263.

9 J. C. Morris, D. L. Callahan, J. Kulik, J. A. Patten and R. Scattergood: J. Am. Ceram. Soc. 78 (1995) 2015.

10 T. P. Leung, W. B. Lee and X. M. Lu: J. Mater. Process. Technol. 73 (1998) 42.

11 P. S. Sreejith and B. K. A. Ngoi: Int. J. Mach. Tools Manuf. 41 (2001).

12 J. W. Yan, K. Syoji and T. Kuriyagawa: J. Mater. Process. Technol. 121 (2002) 363.

13 J. W. Yan, K. Maekawa and J. Tamaki: JSME International Journal Series C Mechanical Systems, Machine Elements and Manufacturing 47 (2004) 29.

14 J. A. Patten, R. Fesperman, S. Kumar, S. McSpadden, J. Qu, M. Lance, R. Nemanich and J. Huening: Appl. Phys. Lett. 83 (2003) 4740.

15 J. A. Patten, W. Gao and K. Yasuto: Journal of Manufacturing Science and Engineering. 127 (2005) 522 .

16 L. Dong and J. A. Patten: Proceedings of the Advanced Laser Applications Conference \& Expo- ALAC 2007 (Advanced Laser Applications Conference, Massachusetts, 2007).

17 P. A. Rebro, F. E. Pfefferkorn, Y. C. Shin and F. P. Incropera: Transactions of the North American Manufacturing Research Institute 30 (2002) 153.

18 D. Ravindra, J. A. Patten and J. Qu: Proc. ASME Int. Manufacturing Science and Engineering Conference 2009 (American Society of Mechanical Engineers, Indiana, 2009) Paper \#84113.

19 A. R. Shayan, H. B. Poyraz, D. Ravindra, M. Ghantasala and J. A. Patten: Proc. ASME Int. Manufacturing Science and Engineering Conference 2009 (American Society of Mechanical Engineers, Indiana, 2009) Paper\# 84207. 